\title{
Estimation of total hepatic blood flow by duplex ultrasound
}

\author{
K M Carlisle, M Halliwell, A E Read, P N T Wells
}

\begin{abstract}
The volume flow rate of blood in the portal vein and the hepatic artery was measured using a duplex ultrasound system. Two sections of the hepatic artery were studied; the common hepatic artery where measurements were made just after the bifurcation of the coeliac axis to splenic and hepatic arteries and the hepatic artery itself, where measurements were made just proximal to the porta hepatis in a straight stretch of artery overlying the portal vein. Total hepatic blood flow was taken as the sum of hepatic artery and portal vein flows. ${ }^{1} A$ group of 10 normal healthy volunteers in the fasting state was studied. The mean (SD) volume blood flow in the vessels was measured to be: hepatic artery $3.5(45 \%) \mathrm{ml} / \mathrm{min} / \mathrm{kg}$, common hepatic artery $6.9(30 \%) \mathrm{ml} / \mathrm{min}^{+} / \mathrm{kg}$, portal vein $13.5(21 \%) \mathrm{ml} / \mathrm{min} / \mathrm{kg}$, total hepatic flow $17 \cdot 0(16 \%) \mathrm{ml} / \mathrm{min} / \mathrm{kg}$.
\end{abstract}

Liver haemodynamics and the changes that occur physiologically and as a result of the presence of liver disease are still largely unexplored problems. Furthermore, such measurements of hepatic blood flow as are possible in human subjects do not allow for the detection of individual changes in portal vein and hepatic artery flow. Thus several accounts of portal vein volume flow measurements using Doppler ultrasound ${ }^{2-5}$ and a few of common hepatic artery measurements ${ }^{67}$ have been published, but there are no reports of total volume flow measurements, comprising portal vein and hepatic artery blood flow. The aim of this study was therefore to try to measure volume flow in hepatic artery by Doppler ultrasound and to allow the estimation of total hepatic blood flow.

We also hoped to show that the ratio of volume flow in the common hepatic artery to the hepatic artery is a constant. A constant ratio of flow in these two parts of the vessel would allow flow measurements in the common hepatic artery to be substituted for flow in the hepatic artery in subjects where visualisation of the hepatic artery is difficult.

Physics \&
Bioengineering, United
Bristol Healthcare Trust
and Department of
Medicine, University of
Bristol, Bristol
K M Carlisle
M Halliwell
A E Read
P N T Wells
Correspondence to:
Dr K M Carlisle, Bristol
General Hospital, Guinea
Street, Bristol BS1 6SY.
Accepted for publication
11 March 1991

\section{Theory}

The volume flow rate of blood in a vessel is given by

$$
\mathrm{Q}=\mathrm{V}_{\text {mean }} \cdot \mathrm{A}
$$

where $Q=$ volume flow, $V_{\text {mean }}=$ mean velocity of blood in vessel, and $A=$ cross sectional area of the vessel. Using a duplex system the cross sectional area (A) is determined from the B-mode or $M$ mode images of the vessel. The mean velocity can be determined by two methods, either from the power spectrum of the returned Doppler signal which relies on even insonation of the vessel by the ultrasound beam or from the maximum velocity.

\section{POWER SPECTRUM}

When a vessel is uniformly insonified by the pulsed ultrasound beam the power spectral density of the returned Doppler signal directly represents the distribution of red cell velocities in the sample volume. ${ }^{89}$ Thus the normalised first moment of the Doppler power spectrum is proportional to the average red cell velocity in the vessel encompassed by the sample volume*

$$
f=\frac{\int^{f m a x} f_{i} \cdot A_{i}^{2} \cdot d f}{\int^{f m a x} A_{i}^{2} \cdot d f}
$$

where $f=$ mean frequency and $A_{i}=$ amplitude of the power spectrum component $f_{i}$.

With a typical duplex scanner, approximately even insonation of the vessel can be obtained in vessels of small diameter. Therefore the mean velocity was calculated from the first moment of the power spectrum for the common hepatic artery and the hepatic artery.

\section{MAXIMUM VELOCITY}

The mean velocity can also be calculated from the maximum velocity if the velocity profile across the vessel is known. For parabolic flow the $\mathrm{V}_{\text {mean }}$ is half the $\mathrm{V}_{\text {max }}{ }^{1011}$ Using Doppler ultrasound the maximum velocity is given by

$$
\mathrm{V}_{\max }=\frac{\mathrm{f}_{\mathrm{dmax}} \cdot \mathrm{C}}{2 \mathrm{f} \cdot \cos \Theta}
$$

where $f_{d \max }=$ maximum Doppler shift frequency, $c=$ speed of sound in soft tissue, $\theta=$ angle of insonation of the beam to the blood flow, and $f=$ transmitted frequency of the ultrasound.

The velocity profile in the portal vein is not parabolic. However, the mean velocity in a venous vessel has been shown experimentally to be $0.57 \mathrm{~V}_{\text {max. }}{ }^{2}$ This was used for the portal vein where complete insonation of the vessel is not possible because of pulse length and sample volume limitations.

\section{Method}

Duplex pulsed Doppler flow measurements were made in the portal vein, hepatic artery, and common hepatic arteries. Initially a group of $\mathbf{4 0}$ normal subjects were scanned to determine a subset in whom the hepatic artery was visualised and who we were confident had no accessory hepatic arteries. This subset consisted of 10 normal women, mean age 32 years (range 21-55 years) and mean weight $54 \mathrm{~kg}$ (range $45-63 \mathrm{~kg}$ ). 
Figure 1: Oblique ultrasound image showing portal vein $(P V)$ and hepatic artery $(P H A)$ just before entry through the porta hepatis. The Doppler cursor is placed in the proper hepatic artery lumen parallel to the portal vein lumen. Beam line and direction of the flow in the proper hepatic artery intersect at an angle of $46^{\circ} . I V C=$ inferior vena cava.

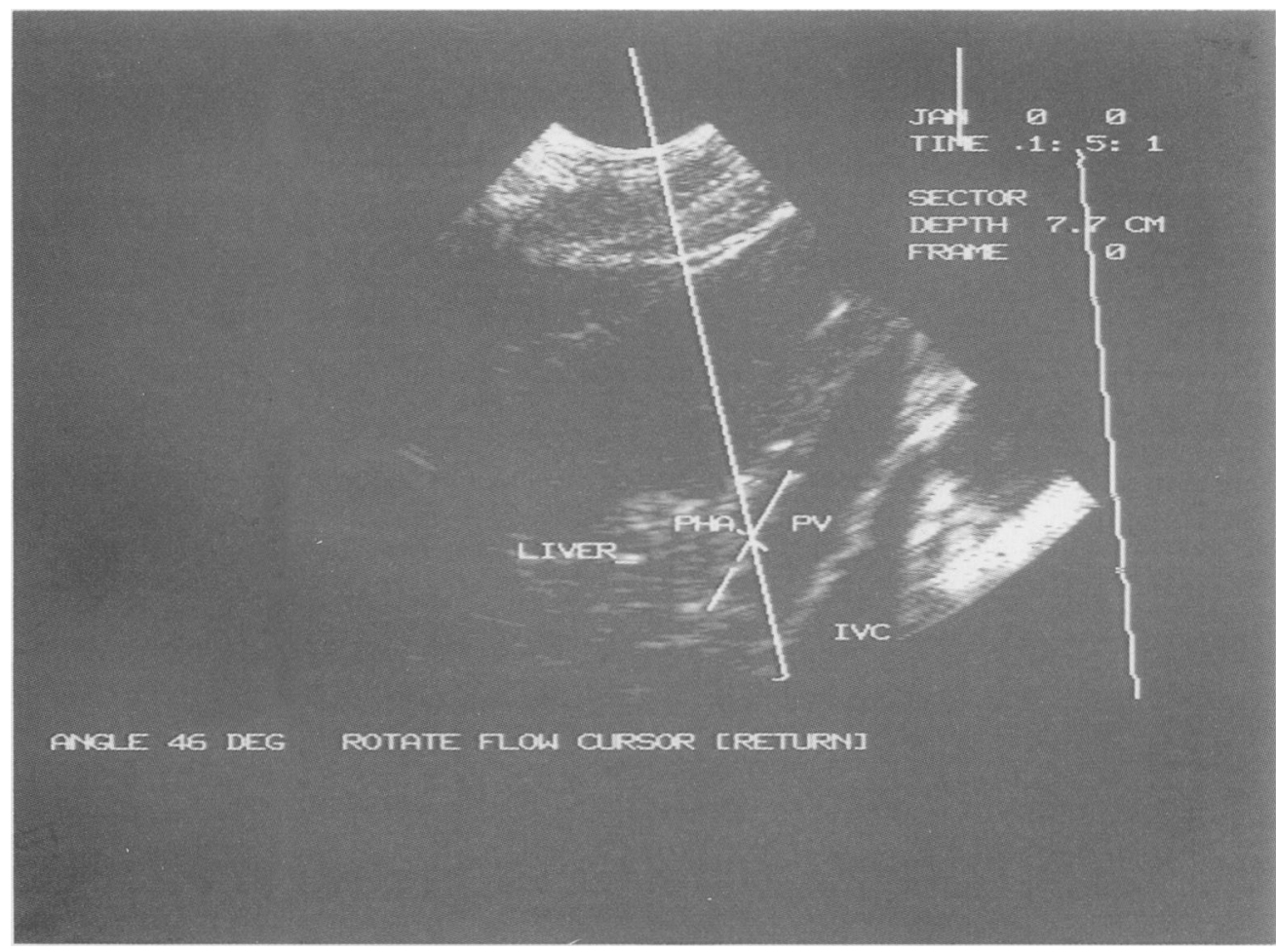

All subjects were studied resting, recumbent, and after overnight fasting.

An ATL 600 duplex pulsed Doppler system with $3 \mathrm{MHz}$ mechanical sector probe was used to measure frequency shift and angle of insonation in the portal vein, common hepatic artery, and hepatic artery. The angle $\theta$ was estimated by aligning a cursor on the frozen image with the direction of the lumen of the vessel. The frequency shift spectra were stored on a microcomputer and by entering the angle $\theta$ mean and maximum velocities were calculated by the software.

A longitudinal image of the portal vein was obtained over the inferior vena cava just after the confluence of the splenic and superior mesenteric veins. The sample volume cursor was placed in the centre of the vessel lumen. The hepatic artery measurements were taken where a straight stretch runs parallel to the portal vein before entry through the porta hepatis (Fig 1). This ensured that measurements were made distal to the point where gastroduodenal and gastric arteries have branched so that only hepatic blood flow was being measured. The common hepatic artery measurements were taken just after the division of coeliac axis and where possible before the gastroduodenal and gastric arteries branched.
Figure 2: Ultrasound image of portal vein $(P V)$ and hepatic artery $(P H A)$ in cross section shown extrahepatically over the inferior vena cava. The callipers measure

anteroposterior diameter of portal vein lumen. Both anteroposterior and lateral measurements are required for cross sectional area calculations.

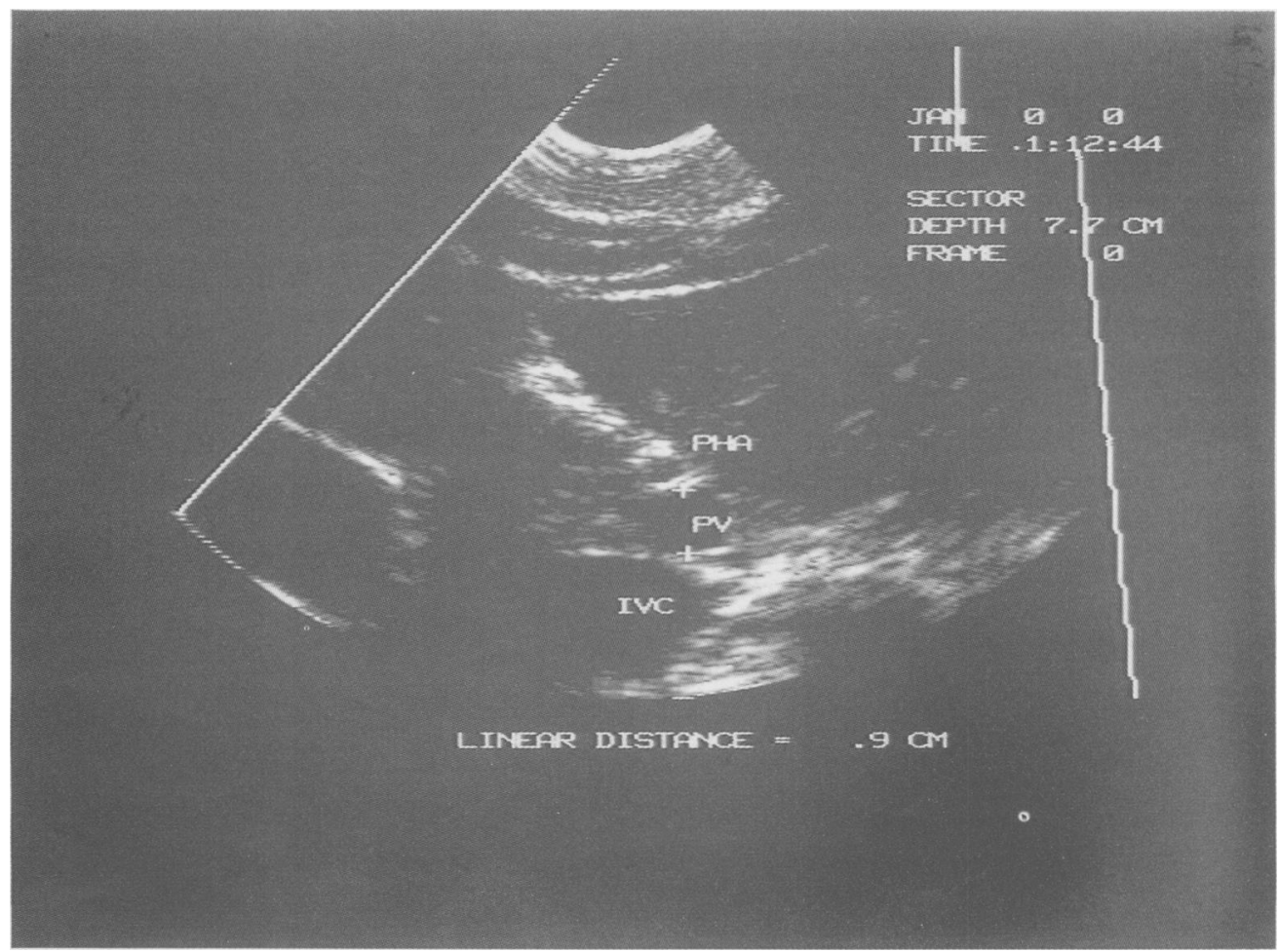


Since velocity measurements are dependent on the angle of insonation $\theta$ between beam and flow direction, measurements were taken where possible at angles of less than $55^{\circ}$ to minimise the errors. Satisfactory angles were most difficult to obtain in the common hepatic artery where the vessel was almost perpendicular to the beam.

Day to day reproducibility of the technique was assessed by taking measurements in the same subject from the same site of each vessel on three separate days under the same physiological conditions.

Interobserver variation between the two observers was determined by taking the mean flow measured by each observer in each subject and obtaining the percentage difference for each vessel.

Two methods were used to determine the vessel diameters required to calculate cross sectional area of the vessels:

(1) By B-mode imaging of the vessel in cross section at the point of Doppler insonation. The gain was reduced and the image size made as large as possible to improve resolution. The callipers of the scanner were placed at the edges of the frozen image of the cross-section of the vessel to determine the diameter across the centre of the vessel both in anteroposterior and lateral directions (Fig 2).

The common hepatic artery and the hepatic artery can be assumed to be cylindrical so only the anteroposterior diameter was required, but the portal vein is normally elliptical in shape so that both anteroposterior and lateral diameters are required.

(2) Changes in the vessel diameter may occur in the portal vein due to transmitted pulsation from the inferior vena cava situated directly behind it and in the hepatic artery due to cardiac pulsations. Thus the second method made use of hard copy of M-mode images of the anteroposterior diameter taken over several cardiac cycles. Averaging the diameter over several cardiac cycles, however, gave agreement of $\pm 0.5 \mathrm{~mm}$ with the instantaneous diameter measured using the callipers. Therefore, as long as Doppler shift and area measurements were averaged over several cardiac cycles the callipers were sufficiently accurate for measuring anteroposterior diameters. In the portal vein M-mode cannot be used to obtain lateral measurements.

The B-mode resolution therefore determines the accuracy of area calculations. For anteroposterior measurements this is $\pm 1 \mathrm{~mm}$ and depends on pulse length. For lateral measurements resolution is $\pm 2 \mathrm{~mm}$ and is a function of beam width. ${ }^{3}$

\section{In vitro calibration}

The values of flow calculated by the software from both $\mathrm{V}_{\text {mean }}$, using the first moment of the power spectrum, and $\mathrm{V}_{\max }$ were compared to measured flows using a flow rig. The fluid used in the experiments was a mixture of water, glycerol, and Sephadex, ${ }^{312}$ chosen to give ultrasonic backscattered characteristics similar to blood. The flow rig comprised a constant head reservoir connected to a horizontal tube of known diameter leading to a measuring cylinder for timed collection of the flow. The Doppler probe was positioned at a constant angle. This angle was measured both from the scanner cursor and by geometry. A $9 \mathrm{~mm}$ range gate was then used to obtain spectra in a given pipe at various flow rates. Pipes of diameters ranging from $4 \mathrm{~mm}$ to $15 \mathrm{~mm}$ were studied and the calculated flow rates using the two methods compared to the actual measured flows.

The low frequencies of the backscattered Doppler signal corresponding to low velocity values were lost due to a $100 \mathrm{~Hz}$ filter in the spectrum analyser so flow values were corrected for this loss of low velocity information.

All the calculated flow values were corrected for the difference of the speed of sound in the Sephadex mixture and in blood. The speed of sound in the Sephadex mixture was measured to be $1750 \mathrm{~m} / \mathrm{s}$ and in blood was taken to be 1540 $\mathrm{m} / \mathrm{s}$. In addition, the effect of refraction between the surrounding water bath and the plastic piping and the pipe and the Sephadex mix was taken into account and a refraction correction factor applied for the change in angle of insonation.

\section{IN VITRO RESULTS}

The angle of insonation could be determined to within $\pm 2^{\circ}$.c In the large diameter pipes flow values calculated from $0.5 \mathrm{~V}_{\max }$ corresponded more closely to the real flow than the flow values calculated using the first moment of the power spectrum. The latter values overestimated the flow by at least $12 \%$. This overestimation was due to the incomplete insonation of the pipe giving rise to the low velocity component of the flow profile close to the boundaries of the pipe being lost.

In the $4 \mathrm{~mm}$ pipe the calculated values for both methods were in close agreement with each other and varied by less than $5 \%$ of the real flow. This is well within the experimental error associated with the timed measurement of the flow.

\section{IN VIVO RESULTS}

Interobserver variation was found to be $7 \%$ in the hepatic artery, $10.5 \%$ in the common hepatic artery and $8 \%$ in the portal vein. The percentage variation from the mean flow for three separate days in the same subject is plotted for each of the vessels. The most reproducible results were obtained in the hepatic artery and portal vein having percentage variations of 3.6 and 2.7 respectively. In the common hepatic artery this was $16 \%$.

\section{Mean velocity}

Mean (SD) velocity for each subject was plotted (Figs 3 and 4). Since the values for $\theta$ did not exceed $55^{\circ}$ the greatest random error in a velocity measurement was $5 \%$. The results of the in vitro calibration showed that the mean velocities calculated from the first moment of the power spectrum in the hepatic and common hepatic arteries must be corrected for the systematic error due to the high pass wall thump filter causing loss of low frequencies. This correction 


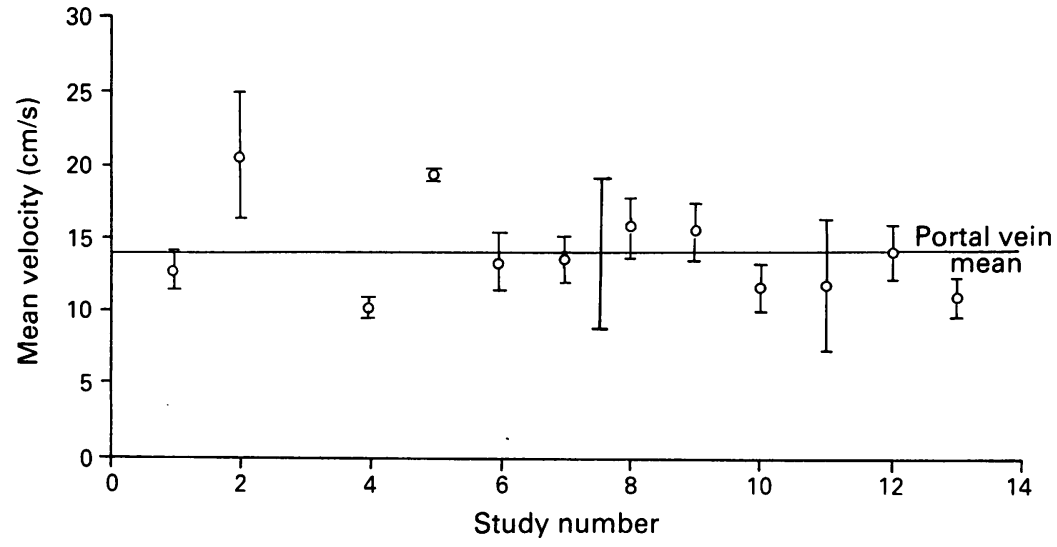

Figure 3: Mean (SD) velocity in the portal vein shown for each subject. Central bar represents mean velocity of the group.

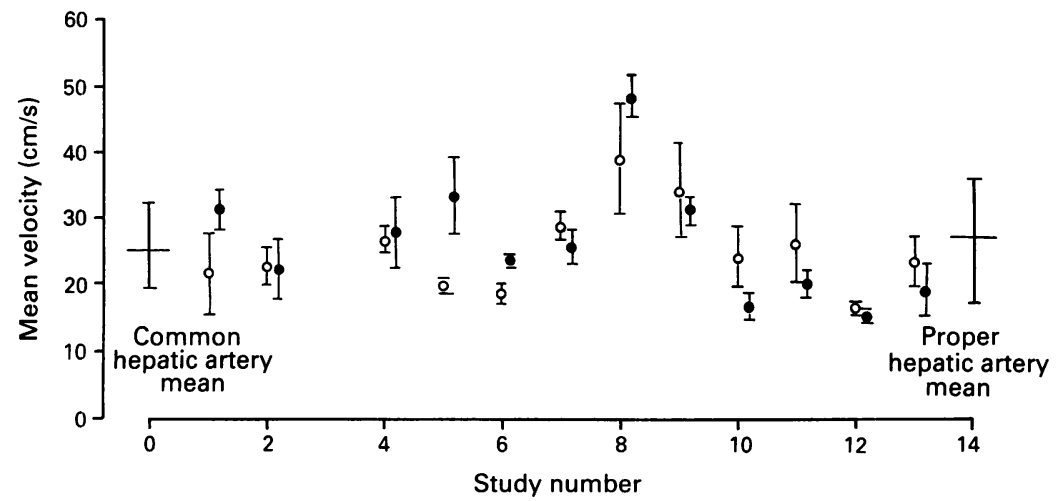

Figure 4: Mean $(S D)$ velocities in the common hepatic artery $(O)$ and proper hepatic artery ( for each subject. Means $(S D)$ for the whole group shown.

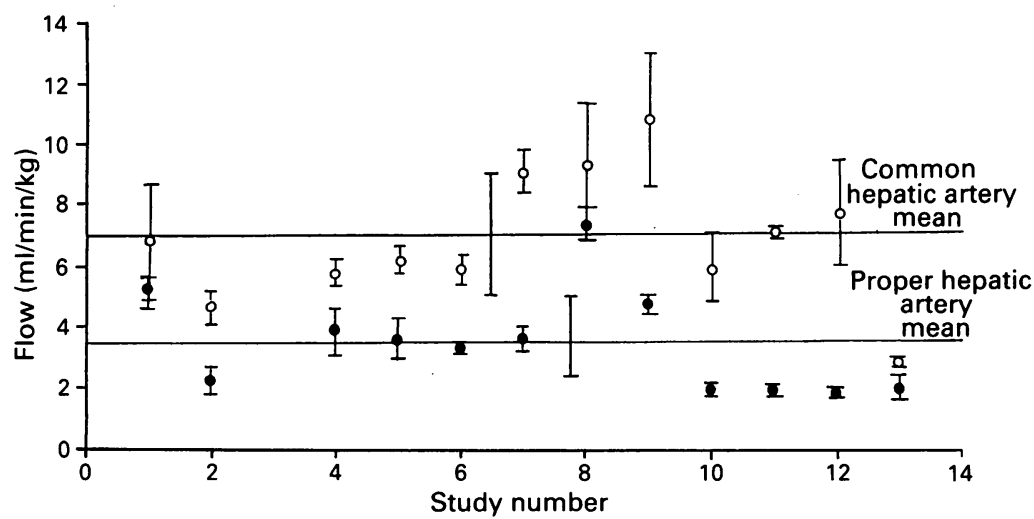

Figure 5: Mean (SD) flow in each subject normalised for body weight for both common hepatic artery $(O)$ and proper hepatic artery $(\mathbf{O})$. Study numbers 5, 6, 7 represent data in the same subject on three consecutive occasions.

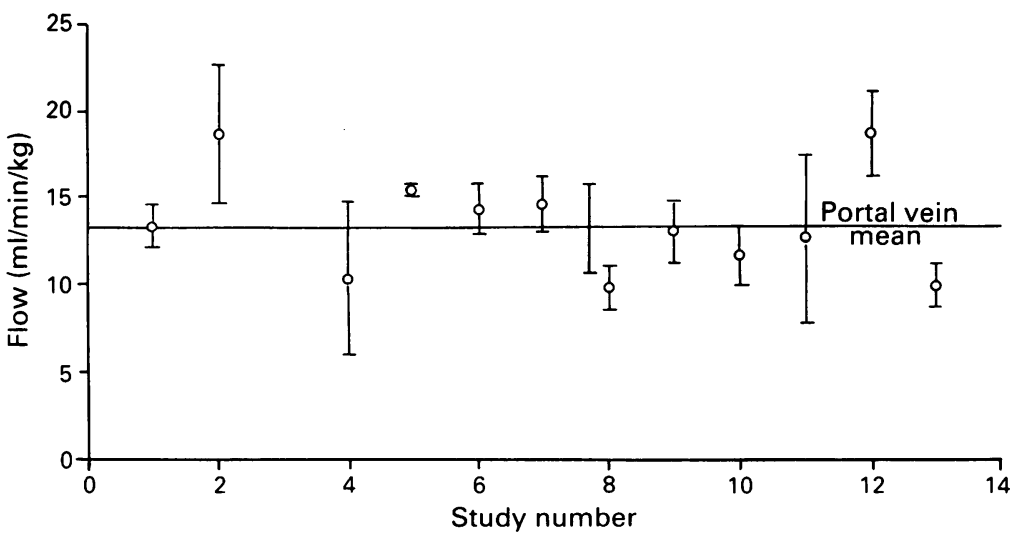

Figure 6: Mean (SD) flow in each subject normalised for body weight in the portal vein. Study numbers 5, 6, 7 represent flow data in the same subject. was particularly important in the hepatic artery, where flow values were low. The use of the first moment of the power spectrum to calculate mean velocities was most appropriate in the hepatic and common hepatic arteries since in pulsatile flow the velocity profile cannot be assumed to be parabolic.

\section{Volume flow}

Volume flow was normalised for weight for each subject to allow comparison of subjects with different liver volumes. Figures 5 and 6 show the mean (SD) flow for each subject studied for different vessels. Figure 5 compares the hepatic and common hepatic arteries and Figure 6 shows the portal vein. The total hepatic flow calculated by summing mean portal vein flow and mean hepatic artery flow in each subject is shown (Fig 7 ). The mean values for the group are given in the Table.

The errors due to the resolution of the spectrum analyser in the computer were calculated to be less than $6 \%$.

We had hoped to show that the ratio of common hepatic to hepatic artery flow was constant, so that a constant fraction of common hepatic artery flow might be used instead of hepatic artery flow, thus extending the population in whom the technique can be used; however, the scatter on the graph shown in Figure 8 does not allow this assumption to be confirmed and ties in with the poor reproducibility already found for common hepatic artery measurements.

\section{Discussion}

In vitro measurements have shown that the duplex system is accurate and reproducible. To apply this method to clinical practice, however, the errors and limitations that arise from body configuration, variable arterial anatomy, and the methodology of the Doppler measurement must be taken into account.

Volume flow measurements in the hepatic artery and portal vein were possible for only a limited selection of the normal population those who are thin and in whom visualisation of both vessels is possible. This applies equally to the male and female populations. Of the male volunteers available, however (who were a small fraction of the total volunteer population), none was of suitable stature to be included in the study. Visualisation depends not only on body habitus but also on the anatomical configuration of the vessels. With the present technique a straight stretch of the hepatic artery is required to determine the angle of insonation $\theta$. Accuracy of angle measurement is essential for mean velocity calculation and thus for volume flow measurement. Where the hepatic artery spirals or changes direction, visualisation is not good enough to determine the angle even if the received Doppler shift signal is satisfactory. In addition, the study population was limited to those in whom the position of gastric and gastroduodenal artery branches could be identified. There will also be a proportion of the population who have variations of the hepatic artery, 


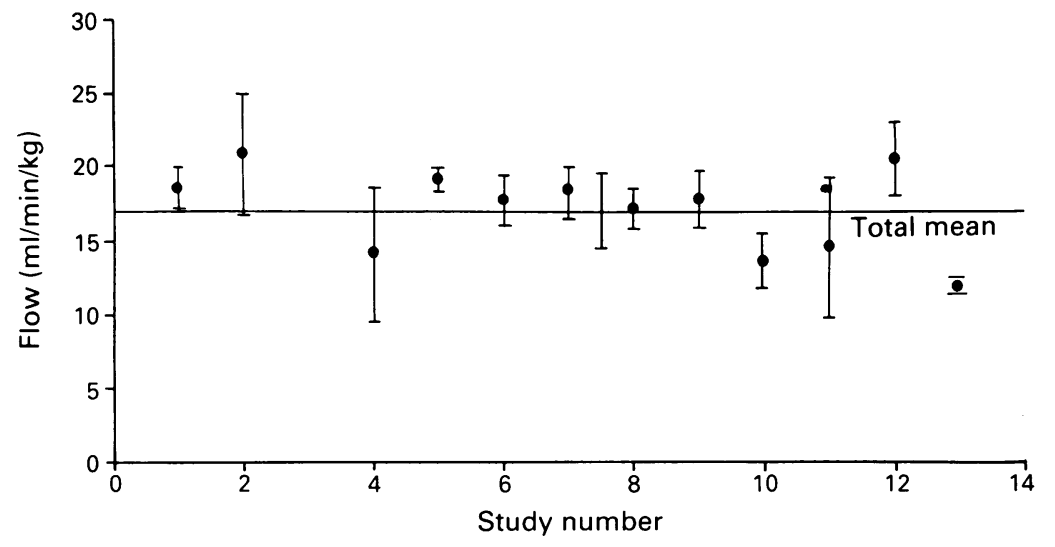

Figure 7: Mean (SD) total hepatic flow in each subject formed from sum of proper hepatic artery and portal vein flow. Mean (SD) total flow is $17 \mathrm{ml} / \mathrm{min} / \mathrm{kg}$.

including accessory right hepatic artery arising bifurcation of hepatic artery, and right or left from the origin of left gastric artery, proximal hepatic artery arising from the coeliac or superior mesenteric artery. These additional branches must be identified and if possible the flow in them measured before total hepatic flow can be calculated.

We are confident that none of the subjects studied had any major variability in arterial blood supply to the liver and where such variability is a possibility the technique of colour Doppler flow imaging should identify these subjects and may possibly allow measurement of anomalous flow so that hepatic arterial flow might still be calculated. Though all subjects had to be thin for study, the deficiency of body fat seen in some patients with parenchymal liver disease may also make these subjects suseptible to study.

The weight normalised values for flows in the common hepatic artery and portal vein fell within the ranges found by other groups ${ }^{2-6}$ and the values for the hepatic artery were within expected physiological ranges. ${ }^{13}$ The day to day reproducibility in the portal vein and hepatic artery were good but the difficulties of obtaining good flow results in the common hepatic artery with this technique were reflected in the poor day to day reproducibility of $16 \%$ and poor interobserver variation of $10 \cdot 5 \%$. The main limitations being that of obtaining a good Doppler angle from the same site with the mechanical sector probe when a linear array probe, not available to us, would have been more appropriate. Since the study was aimed at measuring hepatic artery flow and the measurement of common hepatic artery flow was only implemented to try to increase the study population, these results were not considered detrimental.

The errors associated with the Doppler measurement of absolute volume flow are large. The greatest source of error is in the cross sectional area measurement. This error is determined by the system resolution. For a $3 \mathrm{MHz}$ system this is approximately $1 \mathrm{~mm}$ in the direction of the ultrasound beam. It also depends on the accuracy of the measuring technique. There is little which can improve the resolution due to the beam width in the lateral direction, approximately $2 \mathrm{~mm}$, although reducing the gain improves the effective beam width. In the hepatic artery the vessel can be assumed to be circular and therefore only anteroposterior diameter need be considered. In practice, vessel diameters can be measured to $\pm 1 \mathrm{~mm}$ leading to errors of $\pm 17 \%$ in the portal vein cross sectional area and $\pm 50 \%$ in the hepatic artery cross sectional area (see Table). These errors are inherent and therefore systematic, in addition to the random errors associated with calliper accuracy.

In the portal vein there is a fluctuation observed in the measured velocity which occurs at the cardiac rate. The origin of this fluctuation is not clear. It may well be due to a combination of factors which are likely to include a mechanical change in the area of the portal vein and a change in the downstream impedance of the liver. There is a small change in the anteroposterior diameter of the portal vein, presumably due to mechanical pulsations transmitted from the inferior vena cava. It is not known if the impedance of the liver undergoes cyclic alterations. The accuracy of portal vein volume flow measurements are improved by averaging the area over several cardiac cycles; however, in practice it is only possible to average the anteroposterior diameter using the M-mode and the callipers must be used for lateral measurements.

The combined errors due to area and angle measurement and spectrum analysis give an error in any one measurement of $\pm 20 \%$ for total hepatic flow. The errors associated with area measurement are both random and systematic. Thus the errors in relative flow measurement after a physiological stimulus, for example after feeding, would have a much reduced systematic component. Thus combined errors would be expected to be nearer $\pm 10 \%$.

Thus some of the shortcomings of this noninvasive and repeatable technique are surmountable but problems relating to the existence of an artery that can be scanned, and the limitations resulting from the measurement of small diameter vessels for absolute flow remain.

Even allowing for this it is well recognised that in patients with a variety of liver diseases such as cirrhosis and hepatic tumours, there are significant changes in ratio of portal vein to hepatic artery flow with an increase in the latter in respect to portal venous flow. ${ }^{14}$ Furthermore, there is an increase in total flow produced by this increased arterial component. ${ }^{15}$ At what stage such changes occur and how important they might be diagnostically is unknown. Nor is it

Mean values for the 10 subjects

\begin{tabular}{|c|c|c|c|c|c|c|}
\hline Vessel & $\begin{array}{l}\text { Mean } \\
\text { diameter } \\
(m m)\end{array}$ & $\begin{array}{l}\text { Mean area } \\
\left(\mathrm{mm}^{2}\right)\end{array}$ & $\begin{array}{l}\text { Error in } \\
\text { area }(\%)\end{array}$ & $\begin{array}{l}\text { Mean }(S D) \\
\text { velocity }(\mathrm{cm} / \mathrm{s})\end{array}$ & $\underset{(\mathrm{ml} / \mathrm{min})^{\prime}}{\operatorname{Mean}(S D) \text { flow }}$ & $\begin{array}{l}\text { Mean }(S D) \text { flow } \\
\text { normalised for weight } \\
(\mathrm{ml} / \mathrm{min} / \mathrm{kg})^{\prime}\end{array}$ \\
\hline $\begin{array}{l}\text { Proper hepatic artery } \\
\text { Common hepatic artery } \\
\text { Portal vein } \\
\text { Total hepatic }\end{array}$ & $\begin{array}{r}4 \\
6 \\
12\end{array}$ & $\begin{array}{r}50 \cdot 3 \\
113 \cdot 1 \\
452 \cdot 4\end{array}$ & $\begin{array}{l}50 \\
34 \\
17\end{array}$ & $\begin{array}{l}26 \cdot 2(8 \cdot 7)(33 \%) \\
25 \cdot 1(6 \cdot 2)(24 \%) \\
14 \cdot 2(5 \cdot 4)(38 \%)\end{array}$ & $\begin{array}{l}212(74 \cdot 5)(35 \%) \\
359(126 \cdot 3)(35 \%) \\
724 \cdot 2(161 \cdot 6)(22 \%) \\
940(178)(19 \%)\end{array}$ & $\begin{array}{c}3 \cdot 5(1 \cdot 6)(45 \%) \\
6 \cdot 9(2 \cdot 1)(30 \%) \\
13 \cdot 5(2 \cdot 92)(21 \%) \\
17 \cdot 0(2 \cdot 72)(16 \%)\end{array}$ \\
\hline
\end{tabular}




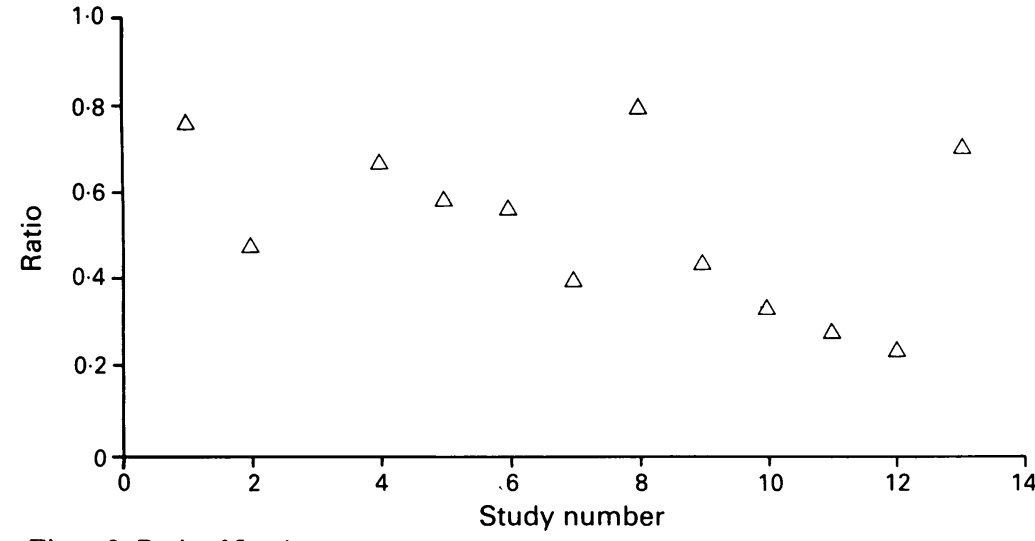

Figure 8: Ratio of flow in common hepatic artery to hepatic artery for each subject.

certain how this change in hepatic haemodynamics might upset the normal variation in in this application may well extend the population for whom the technique is possible.

The technique at present would not be suitable for routine clinical practice, however, it should be possible in suitable subjects with this repeatable method to study diagnostic and prognostic values of changes in hepatic artery portal vein flow and their combination to total flow. This may provide valuable haemodynamic information in a wide variety of acute and chronic hepatic disorders.

1 Netter FH. Digestive System. Ciba Collection. Summit NJ: Ciba, 1957: 3 part 3.

2 Moriyasu F, Nobuyuki B, Osamu N, et al. Clinical application of an ultrasonic duplex system in quantitative measuremen of portal blood flow. $\mathcal{F}$ Clin Ultrasound 1986; 14: 579-88.

3 Brown HS, Halliwell M, Qamar M, Read AE, Evans JM Wells PNT. Measurement of normal portal venous blood flow by Doppler ultrasound. Gut 1989; 30: 503-9. portal venous and hepatic artery flow resulting from physiological stimuli, information which this technique may provide.

In conclusion, present methods of separate flow measurement in the vessels supplying the liver are invasive. They include multiple vascular catheterisation techniques ${ }^{16}$ and implantation of electromagnetic flowmeters. ${ }^{17}$ The two methods of non-invasive flow measurement developing at present are duplex ultrasound and magnetic resonance imaging. There is a report of portal vein flow using magnetic resonance imaging ${ }^{18}$ but none as yet of hepatic artery flow. Thus duplex ultrasound as described above provides the only non-invasive method of flow measurement available for determination of the separate components of the hepatic blood supply.

Duplex ultrasound allows measurement of both the arterial and venous hepatic blood supply. Mean flow rates have been obtained for normal fasting adults in the accepted physiological range. If the population in whom the technique is applicable can be extended its clinical application would be manifold. Reduction of the systematic errors associated with Doppler flow measurement would be possible by making sequential relative measurements. The use of colour Doppler flow imaging 